Biochimica et Biophysica Acta, 497 (1977) 14-28

(C) Elsevier/North-Holland Biomedical Press

BBA 28157

\title{
ANALYSIS OF THE CARBON-13 AND PROTON NMR SPECTRA OF BOVINE CHROMAFFIN GRANULES
}

\author{
ROBERT R. SHARP and EDWARD P. RICHARDS \\ Department of Chemistry, University of Michigan, Ann Arbor, Mich. 48104 (U.S.A.)
}

(Received August 3rd, 1976)

\section{Summary}

Natural abundance carbon-13 and proton NMR spectra of bovine chromaffin granules have been obtained and analyzed using computer simulation techniques. High resolution spectra show the presence of a fluid aqueous phase containing epinephrine, ATP and a random coil protein. The protein spectrum contains unusually intense resonances due to glutamic acid and proline and has been simulated satisfactorily using the known amino acid composition of chromogranin A.

The lipid phase of chromaffin granules gives rise to intense, but very broad, resonances in the carbon- 13 spectrum. Protons in the lipid phase are also observable as a very rapid component of the proton-free induction decay $\left(T_{2} \cong 15\right.$ $\mu \mathrm{s})$.

Linewidths of the carbon-13 spectra have been used to set upper limits on rotational correlation times and on the motional anisotropy in the aqueous phase. These limits show that the aqueous phase is a simple solution (not a gel) that is isotropic over regions much larger than solute dimensions. No gel transition is observed between -3 and $25^{\circ} \mathrm{C}$. The carbon-13 spectra are definitely inconsistent with a lipoprotein matrix model and chromaffin granules previously proposed by Helle and Serck-Hanssen ((1975) Mol. Cell. Biochem. 6, 127-146). Relative carbon-13 intensities of ATP and epinephrine are not consistent with the known 1:4 mol ratio of these components. This fact suggests that epinephrine and ATP are not directly complexed in intact chromaffin granules.

\section{Introduction}

NMR provides a powerful non-perturbing probe of the internal structure of membrane-bound vesicles. Spectral linewidths in NMR depend in a sensitive manner on the details of molecular motion as well as on the anisotropy of the 
local molecular environment, and this dependence can be translated into information about the physicochemical phase structure of intact vesicles. In this and a subsequent paper, NMR linewidths and relaxation times have been used to study the physical chemistry of isolated chromaffin granules. The results allow a critical test of various hypotheses concerning the mechanism of epinephrine storage and release. In particular they offer an experimental basis for assessing the role of the chromogranins, a group of highly soluble non-enzymatic proteins of uncertain function, in the storage mechanism.

Proton $(220 \mathrm{MHz})$ and ${ }^{31} \mathrm{P}(31 \mathrm{MHz})$ spectra of bovine chromaffin granules at $25^{\circ} \mathrm{C}$ have recently been published by Daniels et al. [1]. Their high resolution proton spectra exhibited resonances due to epinephrine and ATP, which are present in concentrations of about 0.50 and $0.125 \mathrm{M}$, respectively, in the vesicles, as well as characteristic protein resonances, presumably due to chromogranin. They concluded that these components are present in "jelly" or "structured gel" phase in the granule interior. They also suggested that chemical shifts in the ${ }^{31} \mathrm{P}$ spectra of ATP are consistent with a binary epinephrine ATP complex, which could function as the storage form of epinephrine.

In this paper we report the carbon- 13 spectra of bovine chromaffin granules at 3 and $25^{\circ} \mathrm{C}$. These and the $100 \mathrm{MHz}$ proton spectra have been analyzed, using computer simulation techniques, in terms of the known biochemical composition of the vesicles. The NMR data place a number of constraints on physicochemical models of the intact vesicles and provide a framework for evaluating proposed mechanisms of epinephrine storage and release. In general, our data are not explained by previous models of chromaffin granule structure, and our interpretation of the NMR spectra is also at variance in certain respects with the conclusions of Daniels et al. [1]. In order to clarify this situation and to provide a condensed and unified theoretical perspective, we give below a brief summary of the factors that determine NMR linewidths in different physicochemical phases.

\section{Influence of phase structure on NMR linewidths}

NMR linewidths of spin-1/2 isotopes such as protons and ${ }^{13} \mathrm{C}$ result from magnetic dipole couplings between neighboring nuclei. Intrinsic linewidths are determined by the time scale (characterized by a correlation time $\tau_{c}$ ) over which dipolar couplings are averaged to zero by Brownian motion. In heterogeneous media they are also very sensitive to anisotropy in molecular tumbling that prevents complete averaging of dipolar couplings. It is this sensitive dependence of linewidth on $\tau_{c}$ and on the presence of long range molecular order that provides a useful probe of physicochemical phase structure. The theory of NMR linewidths in crystalline solids and isotropic liquids is the subject of an extensive literature that is reviewed in references $14-16$. Linewidths in interfacial systems [17-21], in gels [22-27] and in lipid bilayers [28-30] have also been analyzed both theoretically and experimentally during the last 15 years.

(1) Polycrystalline solids. In this limit the lineshape is a very broad envelope of transitions due to the fact that the local magnetic field at which individual spins come into resonance varies rapidly from point to point throughout the solid lattice. Each nucleus resonates in a magnetic field that is the sum of a large 
external field $H_{0}$ and a much smaller local field that results from the static distribution of nuclear magnetic dipole moments. If each nucleus were coupled magnetically to a single neighbor in the lattice, resonance would occur at two values of the external field, corresponding to the two orientations of the neighboring spin $\left(I_{z}= \pm 1 / 2\right)$. Thus, resonance would occur as a doublet, at the two field values

$H=H_{0} \pm \frac{3 \gamma \hbar\left(1-3 \cos ^{2} \theta\right)}{4 r^{3}}$

The doublet splitting depends on the angle $\theta$ between the internuclear vector $r$ and the external field direction, and on the gyromagnetic ratio $\gamma$. In a polycrystalline solid the resonance is an envelope of transitions, the shape of which may be calculated by summing over a random distribution of neighboring spin states and integrating over all orientations of the solid lattice $[15,16]$. The lineshape from a solid sample is in general complex and has a characteristic width of order $40 \mathrm{kHz}$. Thus, linewidths from solids are typically $10^{3}-10^{6}$ times greater than those from mobile liquids.

(2) Liquids and plastic solids. Brownian motion in these phases causes the local dipolar field to fluctuate rapidly due to fluctuations in $r$ and $\theta$. Line narrowing occurs when the motional correlation time $\tau_{c}$ is less than or comparable to the inverse of the rigid lattice linewidth, i.e. $\tau_{\mathrm{c}}<10^{-5} \mathrm{~s}$. When $\tau_{\mathrm{c}}$ is shorter than this value, the lineshape becomes Lorentzian and its width decreases monotonically with decreasing $\tau_{\mathrm{c}}$.

To a good approximation in the present experiments, dipolar interactions of protons in the aqueous phase are dominated by intramolecular proton-proton couplings, since deuteration of the solvent effectively suppresses the intermolecular dipolar contribution. Neglecting the intermolecular term, the motional contribution to the linewidth in an isotropic liquid is given by ref. 15

$$
W=\left(\frac{5}{4 \pi}\right) \gamma^{4} \hbar^{2} \sum_{k} r_{j k}^{-6}\left\{\tau_{\mathrm{R}}+\frac{5}{3} \frac{\tau_{\mathrm{R}}}{1+\omega_{\mathrm{H}}^{2} \tau_{\mathrm{R}}^{2}}+\frac{2}{3} \frac{\tau_{\mathrm{R}}}{1+4 \omega_{\mathrm{H}}^{2} \tau_{\mathrm{R}}^{2}}\right\}
$$

where $\omega_{\mathrm{H}}$ is the angular Larmor frequency of protons, and $\tau_{\mathrm{R}}$ is the molecular reorientational correlation time. This expression describes the effect of isotropic molecular reorientation only and neglects linewidth contributions due to motional anisotropy that may occur in heterogeneous media. In computing the analogous component of ${ }^{13} \mathrm{C}$ linewidths, it is valid to assume that the linewidths of protonated carbons are strongly dominated by dipolar couplings to directly bonded protons, since $W$ varies as $r^{-6}$. In this approximation the ${ }^{13} \mathrm{C}$ linewidth is

$W=(20 \pi)^{-1} \hbar^{2} \gamma_{\mathrm{C}}^{2} \gamma_{\mathrm{H}}^{2} N r_{\mathrm{CH}}^{-6} N f\left(\tau_{\mathrm{R}}\right)$,

$f\left(\tau_{\mathrm{R}}\right)=4 \tau_{\mathrm{R}}+\frac{\tau_{\mathrm{R}}}{1+\left(\omega_{\mathrm{H}}-\omega_{\mathrm{C}}\right)^{2} \tau_{\mathrm{R}}^{2}}+\frac{3 \tau_{\mathrm{R}}}{1+\omega_{\mathrm{C}}^{2} \tau_{\mathrm{R}}^{2}}+\frac{6 \tau_{\mathrm{R}}}{1+\left(\omega_{\mathrm{H}}+\omega_{\mathrm{C}}\right)^{2} \tau_{\mathrm{R}}^{2}}+\frac{6 \tau_{\mathrm{R}}}{1+\omega_{\mathrm{H}}^{2} \tau_{\mathrm{R}}^{2}}$

where $N \geq 1$ is the number of directly bonded protons. Eqns. 1 and 2 both require that the medium be isotropic on the scale of molecular dimensions. In heterogeneous media, linewidth measurements provide only an upper limit to rotational correlation times. 
(3) Anisotropic fluids: lipid bilayers and solid-liquid interfaces. These phases are characterized by macroscopic anisotropy that produces preferential orientation of one molecular axis, while permitting rapid rotational motion about other axes. In the presence of motional anisotropy, dipolar couplings average to a non-zero value. Even in the presence of rapid reorientation, nuclear resonances exhibit a broadened envelope with (in general) non-Lorentzian structure, similar to that of a solid lattice. Woessner and Snowden [17-21] have analyzzed ${ }^{1} \mathrm{H}_{2} \mathrm{O}$ signals in interfacial systems, such as Montmorillonite clays, which contain stacked layers of silicate platelets separated by water layers of variable thickness. Molecules in the water layers exhibit considerable motional anisotropy. The residual doublet splitting for a pair of protons that are tumbling anisotropically near an interface is given by ref. 17 .

$\Delta \nu(\mathrm{Hz})=\frac{3}{8 \pi} \gamma^{2} \hbar r^{-3}\left\langle 3 \cos ^{2} \theta^{\prime \prime}-1\right\rangle\left(3 \cos ^{2} \theta^{\prime}-1\right)$

where $\theta^{\prime}$ is the angle between the external field and the normal to the surface, and $\theta^{\prime \prime}$ is the angle between the internuclear vector $r$ and the normal direction. Measured doublet splittings in clay preparations are of the order of $6000 \mathrm{~Hz}$, and values of $\left\langle 3 \cos ^{2} \theta^{\prime \prime}-1\right\rangle$ equal to 0.2 are typical in water layers a few molecules thick. Line broadenings in the clays vary approximately inversely as the thickness of the water layer, since protons diffuse rapidly on the NMR time scale throughout the aqueous phase. Thus, substantial edge effects are observed in NMR spectra even when the fluid phase is considerably larger than molecular dimensions.

\section{Experimental}

Chromaffin granules were isolated from beef adrenals using a procedure developed by Hillarp [2] and Smith and Winkler [3]. The adrenals are removed within a few minutes of death and kept on ice for approx. $1 \mathrm{~h}$ prior to the beginning of isolation procedures. Medullary tissue from 30 adrenals is homogenized in isotonic solution $(150 \mathrm{mM} \mathrm{KCl})$ and centrifuged at low speed $(600$ $X g$ for $10 \mathrm{~min}$ ) to remove coarse cellular debris. The supernatant is then washed several times in isotonic $\mathrm{KCl}$ in ${ }^{2} \mathrm{H}_{2} \mathrm{O}$ and centrifuged at higher speed (20 $000 \mathrm{X}$ $g$ for $20 \mathrm{~min}$ ) in a Sorvall Superspeed centrifuge. The washings remove extravesicular epinephrine and progressively deuterate the solvent. In the initial experiments homogenization was carried out in isotonic sucrose. All procedures were carried out near $3^{\circ} \mathrm{C}$, at which temperature the granules are stable indefinitely with respect to efflux of epinephrine and ATP [4-7].

High resolution NMR spectra were obtained using a JEOL JNM-PFT/100 NMR spectrometer and a JEOL JNM-VT-30 temperature controller. Sample temperature could be maintained within $\pm 1^{\circ} \mathrm{C}$ and was measured using a thermometer in a dummy sample tube. The carbon spectra were referenced to the aromatic epinephrine peaks, using literature values for their chemical shifts [8]. These peaks have the same relative shift in the vesicles as in dilute aqueous solution at $\mathrm{p}^{2} \mathrm{H}$ 7. Proton spectra were referenced to the internal sucrose resonances. 
A free induction decay of protons in the ordered lipid phase of the vesicles was obtained at $3^{\circ} \mathrm{C}$ using a Bruker $\mathrm{B}-\mathrm{KR} 322 \mathrm{~S}$ variable frequency pulsed spectrometer operating at $43.5 \mathrm{MHz}$. The free induction decay was collected on a Hewlett-Packard Model 184 storage oscilloscope.

\section{Results}

The biochemistry of bovine chromaffin granules has been reviewed by Smith [9] and Stjärne [10]. The granules contain a water-soluble fraction that includes catecholamines (20.5\% of dry weight), ATP (15.0 wt.\%), and chromogranins (27 wt.\%) with smaller amounts of ascorbate $(0.46 \mathrm{wt} . \%)$ [11] and inorganic ions. The lipid fraction (22\% of the total dry weight) is particularly rich in cholesterol ( $59 \mathrm{~mol} \%$ ), and includes substantial amounts of lysophosphatidylcholine among its phospholipids. Three enzymes, dopamine- $\beta$-hydroxylase, a $\mathrm{Mg}^{+2}$-dependent ATPase and cytochrome $b-559$, are also associated with the insoluble fraction.

The natural abundance carbon-13 NMR spectrum of isolated chromaffin vesicles at $25^{\circ} \mathrm{C}$ is shown in Fig. 1 . The spectrum was obtained after 30000 scans under random noise proton decoupling conditions. A computer simulation of the spectrum, based on literature assignments of the main water-soluble components of the vesicles (epinephrine, ATP and chromogranin A) are shown in the same figure for comparison. Literature values of the chemical shifts used in this simulation, as well as in the simulations of the proton spectra, are given in Table I.

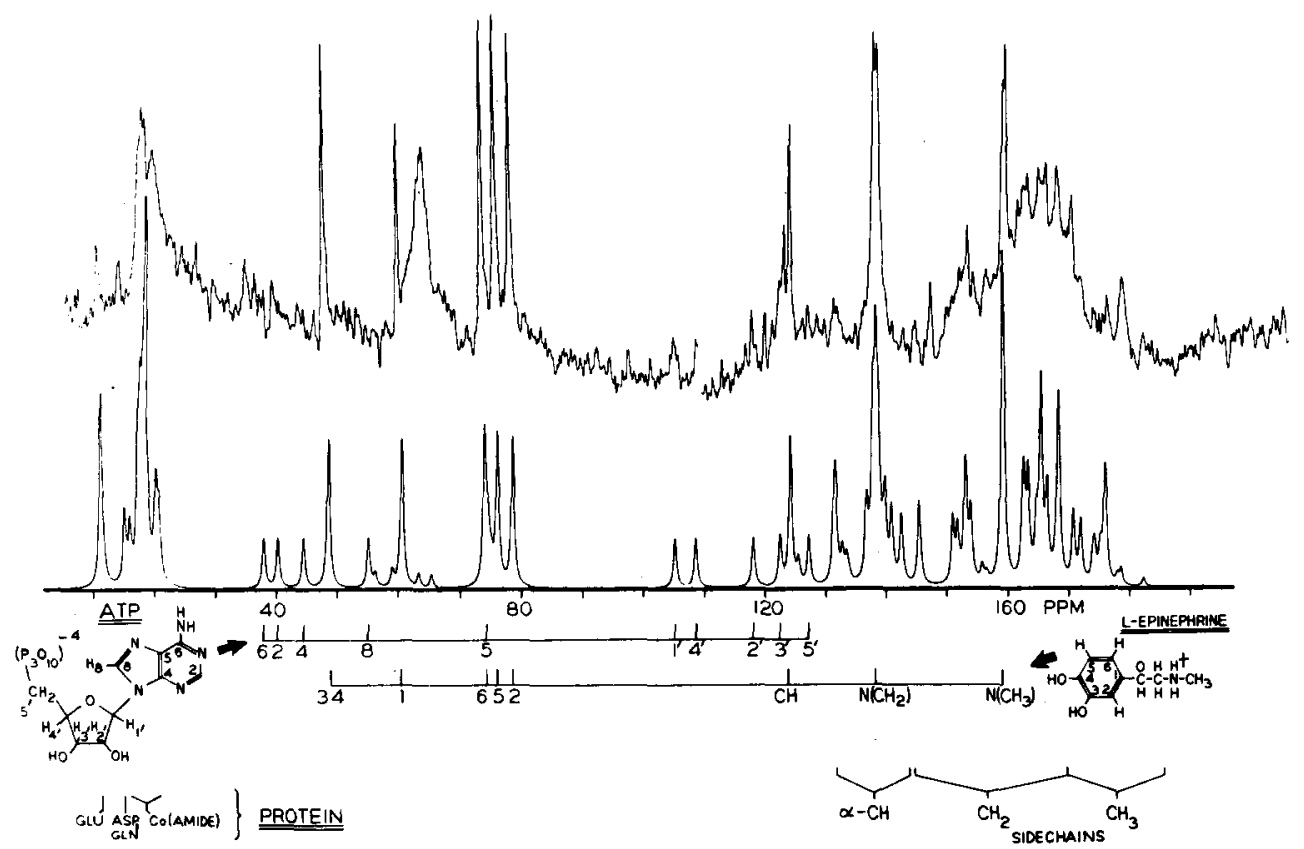

Fig. 1. Natural abundance carbon-13 NMR spectrum (25.1 MHz) of bovine chromaffin granules at $25^{\circ} \mathrm{C}$ in ${ }^{2} \mathrm{H}_{2} \mathrm{O}$; below, computer simulation of the spectrum based on literature assignments of epinephrine [8], ATP [8] and chromogranin (Table I of the text). 
TABLE I

CARBON-13 AND PROTON CHEMICAL SHIFTS OF AMINO ACIDS IN NON-TERMINAL PEPTIDES

Carbon-13 shifts (ppm relative to $\mathrm{CS}_{2}$ ) are taken from references $45-46$ unless otherwise noted. Proton chemical shifts (parts per $10^{8}$ relative to internal DDS) are shown in parentheses and are taken from ref. [48]. The $\alpha\left(\mathrm{C}-{ }^{1} \mathrm{H}\right)$ peptide and titration shifts are estimated using Jardetzky's parameters (ref. 13). The number of amino acids per 77000 molecular weight peptide of chromogranin $A$ is given in parentheses in the first column [9]. DDS, 2,2-dimethyl-2-silapentane-5-sulfonic acid.

\begin{tabular}{|c|c|c|c|c|c|c|}
\hline $\begin{array}{l}\text { Chromogranin } \\
\text { amino acid }\end{array}$ & $\mathbf{c}_{0}$ & $\alpha-\mathrm{CH}$ & $\beta-\mathrm{CH}_{2}$ & $\gamma-\mathrm{CH}_{2}$ & Other & \\
\hline$G l u+G \ln (156)$ & 18.5 & $\begin{array}{l}138.6 \\
(423)\end{array}$ & $\begin{array}{l}165.5 \\
(206)\end{array}$ & $\begin{array}{l}159.4 \\
(233)\end{array}$ & $11.4(\mathrm{Glu})$ & $15.0(G \ln )$ \\
\hline Pro (68) & 17.9 & $\begin{array}{l}131.9 \\
(462)\end{array}$ & $\begin{array}{l}163.5 \\
(207,232)\end{array}$ & $\begin{array}{l}168.4 \\
(201)\end{array}$ & $\begin{array}{l}145.7 \\
(235)\end{array}$ & \\
\hline Lys (57) & 18.6 & $\begin{array}{l}139.0 \\
(424)\end{array}$ & $\begin{array}{l}162.7 \\
(187)\end{array}$ & $\begin{array}{l}170.9 \\
(146)\end{array}$ & $\begin{array}{l}166.6(\delta) \\
(170)\end{array}$ & $\begin{array}{l}153.4(\epsilon) \\
(302)\end{array}$ \\
\hline Asp + Asn (56) & 18.9 & $\begin{array}{l}141.2 \\
(436)\end{array}$ & $\begin{array}{l}154.2 \\
(270)\end{array}$ & & 15.5 (Asp) & 18.0 (Asn) \\
\hline Ser (55) & 20.5 & $\begin{array}{l}137.0 \\
(433)\end{array}$ & $\begin{array}{l}131.6 \\
(393)\end{array}$ & & & \\
\hline Ala (55) & 17.4 & $\begin{array}{l}142.8 \\
(427)\end{array}$ & & & $\begin{array}{l}176.2(\mathrm{Me}) \\
(146)\end{array}$ & \\
\hline Gly (52) & 20.9 & $\begin{array}{l}150.2 \\
(404)\end{array}$ & & & & \\
\hline Leu (50) & 17.8 & $\begin{array}{l}140.1 \\
(421)\end{array}$ & $\begin{array}{l}153.2 \\
(170)\end{array}$ & $\begin{array}{l}168.5 \\
(170)\end{array}$ & $\begin{array}{l}170.6(\mathrm{Me}) \\
(95)\end{array}$ & $\begin{array}{l}172.1(\mathrm{Me}) \\
(95)\end{array}$ \\
\hline $\operatorname{Arg}(42)$ & 18.7 & $\begin{array}{l}139.1 \\
(425)\end{array}$ & $\begin{array}{l}164.9 \\
(187)\end{array}$ & $\begin{array}{l}168.4 \\
(167)\end{array}$ & $\begin{array}{l}152.0(\delta) \\
(322)\end{array}$ & $36.1(\xi)$ \\
\hline Val (25) & 18.8 & $\begin{array}{l}133.0 \\
(410)\end{array}$ & $\begin{array}{l}162.9 \\
(225)\end{array}$ & & $\begin{array}{l}174.4(\mathrm{Me}) \\
(97)\end{array}$ & $\begin{array}{l}175.2(\mathrm{Me}) \\
(102)\end{array}$ \\
\hline Thr (19) & 20.4 & $\begin{array}{l}133.7 \\
(402)\end{array}$ & $\begin{array}{l}125.8 \\
(422)\end{array}$ & & $\begin{array}{l}174.1(\mathrm{Me}) \\
(131)\end{array}$ & \\
\hline $\operatorname{His}^{b}(13)$ & 20.6 & $\begin{array}{l}138.8 \\
(449)\end{array}$ & $\begin{array}{l}165.0 \\
(318)\end{array}$ & & $\begin{array}{l}56.8\left(\mathrm{C}_{2}\right) \\
(784)\end{array}$ & $\begin{array}{l}75.8\left(\mathrm{C}_{4}\right) \\
(708)\end{array}$ \\
\hline Met (13) & 18.7 & $\begin{array}{l}139.8 \\
(434)\end{array}$ & $\begin{array}{l}162.7 \\
(212)\end{array}$ & $\begin{array}{l}163.5 \\
(264)\end{array}$ & $\begin{array}{l}178.7(\mathrm{Me}) \\
(212)\end{array}$ & \\
\hline Phe $^{a}$ (11) & 19.8 & $\begin{array}{l}136.7 \\
(449)\end{array}$ & $\begin{array}{l}155.9 \\
(311,327)\end{array}$ & $56.7(\mathrm{C}-1)$ & $\begin{array}{l}62.6\left(\mathrm{C}_{2,3}\right) \\
(740)\end{array}$ & $\begin{array}{l}64.3\left(\mathrm{C}_{4}\right) \\
(735)\end{array}$ \\
\hline $\operatorname{Tyr}^{a}(8)$ & 19.7 & $\begin{array}{l}138.2 \\
(442)\end{array}$ & $\begin{array}{l}155.3 \\
(285,310)\end{array}$ & $63.2(\mathrm{C}-1)$ & $\begin{array}{l}61.7\left(\mathrm{C}_{2}\right) \\
(719)\end{array}$ & $\begin{array}{l}76.8\left(C_{3}\right) \quad 37.8\left(C_{4}\right) \\
(688)\end{array}$ \\
\hline Ile (7) & 18.9 & $\begin{array}{l}134.1 \\
(415)\end{array}$ & $\begin{array}{l}156.8 \\
(145)\end{array}$ & $\begin{array}{l}168.3 \\
(124)\end{array}$ & $\begin{array}{l}178.0\left(\gamma_{2}\right) \\
(92)\end{array}$ & $\begin{array}{l}182.4(\delta) \\
(99)\end{array}$ \\
\hline $\operatorname{Cys}^{b}(3)$ & 11.7 & $\begin{array}{l}137.3 \\
(446)\end{array}$ & $\begin{array}{l}167.7 \\
(305)\end{array}$ & & & \\
\hline
\end{tabular}

\footnotetext{
a ${ }^{13} \mathrm{C}$ chemical shifts for non-terminal peptides taken from Cristl and Roberts [49].

b ${ }^{13} \mathrm{C}$ chemical shifts taken from Stothers [50] and corrected for peptide shifts using parameters of Cristl and Roberts [49].
}

The intense, relatively narrow $(9-14 \mathrm{~Hz})$ resonances of epinephrine dominate the carbon spectrum. Five peaks representing the six carbons of the catechol moiety appear in the aromatic region (C-3 and C-4 are accidentally degenerate) while the methine, methylene and methyl carbons lie to progressively 
higher field. The latter two are partly obscured by overlapping protein and possibly lipid resonances. Peaks due to ATP are also observable in the spectrum, although they are of surprisingly small amplitude compared to the single carbon resonances of epinephrine. Several of the ATP peaks $\left(\mathrm{C}_{4}, \mathrm{C}_{8}, \mathrm{C}_{5}, \mathrm{C}_{5}\right)$ are indistinguishable from the baseline noise, while several others $\left(\mathrm{C}^{\prime} 1^{\prime}, \mathrm{C}-4^{\prime}, \mathrm{C}-2^{\prime}\right.$, C-3', C-6) are weak but definitely present. The expected ratio of peak amplitudes, assuming equal linewidths and nuclear Overhauser enhancements, is approximately 4 epinephrine: 1 ATP [2]. This ratio is used in the computer simulation. The observed ratio is much greater than $4: 1$ and probably indicates that ATP reorients more slowly than does epinephrine. A longer reorientational correlation time would cause differential broadening of the ATP peaks and consequently lower amplitude for a given total intensity. In addition, if the correlation time of ATP is of order $1.0\left(10^{-9}\right) \mathrm{s}$ or longer, the Overhauser enhancement decreases to unity causing a loss of real intensity. In any event, the unexpectedly low ratio of peak amplitudes of the protonated carbons of ATP relative to epinephrine suggests a difference of correlation times for these molecules. That the actual ratio of ATP and epinephrine concentrations in the aqueous phase is very near $1: 4$ can be shown by comparing integrals in the proton spectra of the resonances of $\mathrm{H}_{2}$ and $\mathrm{H}_{8}$ on ATP with those of the aromatic protons of epinephrine. The proton spectra, which are discussed below, are not subject to Overhauser enhancements and are consistent with a ratio 1 ATP : 3.8 epi, which is within experimental uncertainty of the expected value. (A downfield shoulder on the epinephrine peak is apparent in Fig. 2 and in the spectra of ref. 1; this shoulder is subtracted in the comparison of integrals.)

Much of the remaining intensity consists of protein resonances. This fact is evident from the intense peaks in the region $17-21 \mathrm{ppm}$, which is characteristic of the amide carbon resonances of polypeptides. The protein peaks are quite narrow, generally less than $30 \mathrm{~Hz}$ wide, and correspond to a molecule tumbling rapidly and nearly isotropically in a solution phase (this point is discussed at somewhat greater length below). Thus, the protein peaks are assigned to chromogranin, the main soluble protein component. A simulation of the spectrum, based on the known amino acid composition of chromogranin A [9, 12], gives satisfactory agreement with the observed spectral structure downfield in the amide region and upfield in the methylene-methyl region. Chromogranin is very unusual in its high content of glutamic acid, glutamine, aspartic acid, and asparagine, which together comprise $31 \%$ of all residues. Resonances of the sidechain carboxyls and amide carbons of these residues stand out clearly at 11.5 and $15.5 \mathrm{ppm}$. The insoluble protein fraction is expected to have peaks much too broad to contribute to the high resolution structure in Fig. 1.

Underlying the resolvable structure are two very broad humps centered near 125 and $165 \mathrm{ppm}$. These and the broad, intense peak at $64 \mathrm{ppm}$ do not arise from the soluble components and are probably associated with the ordered lipid phase.

Experimental and simulated $100 \mathrm{MHz}$ proton spectra are shown in Fig. 2. In addition to the large $\mathrm{H}^{2} \mathrm{HO}$ peak at $4.9 \delta$, there is a great deal of partially resolved intensity arising from epinephrine, ATP and chromogranin. Assignment of the peaks is described in the figure. A computer simulation of the 


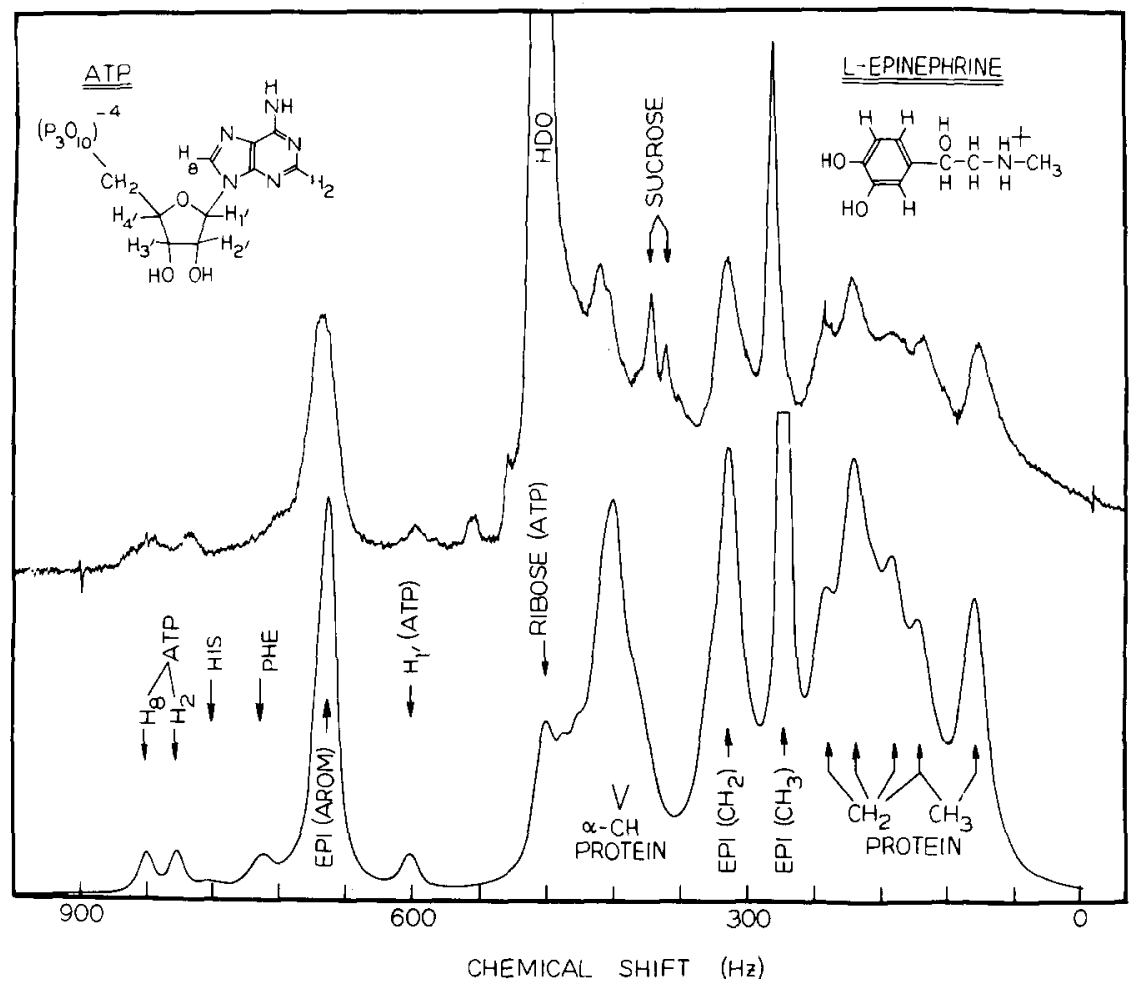

Fig. 2. $100 \mathrm{MHz}$ proton spectrum of chromaffin granules in ${ }^{2} \mathrm{H}_{2} \mathrm{O}$ at $25^{\circ} \mathrm{C}$; below, computer simulation of the proton spectrum.

epinephrine, ATP and chromogranin intensity produces an excellent fit of the experimental spectrum with the exception of two peaks at 3.90 and $3.75 \delta$. These peaks originate from residual sucrose in the sample.

A histogram of the protein amino acid resonances is shown in Fig. 3. A comparison with the experimental spectrum shows that the terminal methyls of leucine, isoleucine and valine comprise a single peak centered at $0.96 \delta$, while the methylene sidechain resonances give rise to four partially resolved peaks to lower field. The abnormally large proportion of glutamic acid and proline in chromogranin is apparent in the two intense methylene peaks at 2.06 and 2.33 ppm. These peaks derive most of their intensity from the $\beta$ and $\gamma$ protons of glutamic acid and proline and consequently are characteristic of the amino acid composition of chromogranin. The backbone $\alpha-\mathrm{CH}$ protons (except for those on glycine) from a single large peak near $4.3 \delta$ that partially overlaps the $\mathrm{H}^{2} \mathrm{HO}$ resonance. Unlike sidechain resonances, the $\alpha-\mathrm{CH}$ protons are sensitive to helix formation in a polypeptide and are shifted upfield by $0.3-0.6 \mathrm{ppm} \mathrm{[13]} \mathrm{in}$ helical regions relative to a random coil peptide. The position of the $\alpha-\mathrm{CH}$ peak at $4.3 \delta$ is consistent with a predominantly random coil form of chromogranin.

Both the carbon-13 and proton spectra have been observed at several temperatures between -3 and $25^{\circ} \mathrm{C}$. Gradual narrowing of the resonances is observed as the temperature is raised from 3 to $25^{\circ} \mathrm{C}$ (see Fig. 4), but no sign of a gel transition, predicted by Smith [9] is apparent. The spectra show very little 


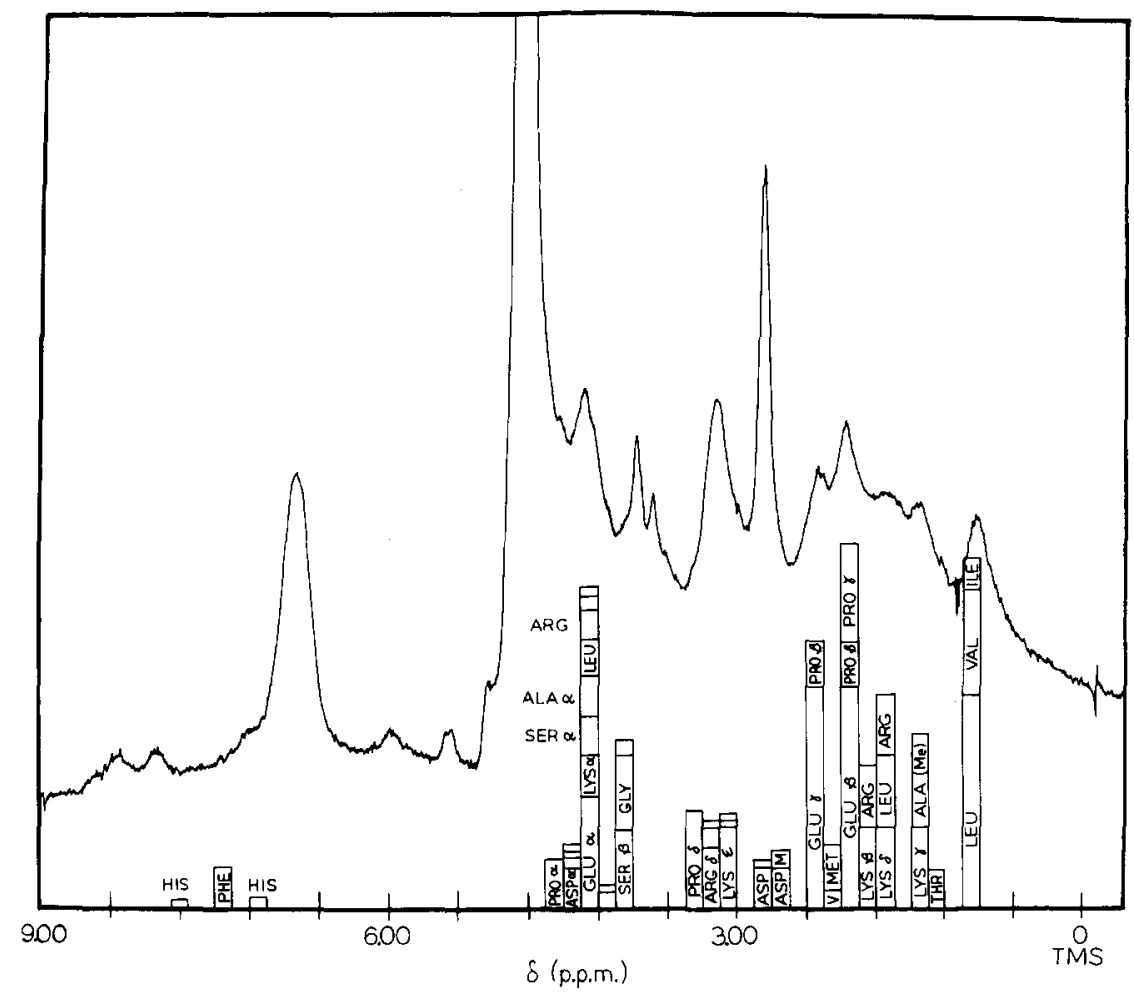

Fig. 3. Comparison of the experimental proton spectrum at $25^{\circ} \mathrm{C}$ and a histogram of amino acid intensities corresponding to the known amino acid composition of chromogranin (Table I of the text).

change with time when the granules are maintained at $25^{\circ} \mathrm{C}$ for $48 \mathrm{~h}$. Recooling the granules to $3^{\circ} \mathrm{C}$ restores most but not all of the original linewidth.

The aqueous phase of chromaffin vesicles

Figs. 1-4 show that the water-soluble components of chromaffin granules (epinephrine, ATP and chromogranin) have quite narrow resonances $\left(\Delta v_{1 / 2} \approx\right.$ $10-40 \mathrm{~Hz}$ ) which are consistent only with a fairly fluid, essentially isotropic, environment. Unfortunately, the two parameters that characterize motional fluidity and anisotropy, namely $\tau_{R}$ and $\left\langle 3 \cos ^{2} \theta^{\prime \prime}-1\right\rangle$, cannot be calculated individually from linewidth measurements alone. It is possible, however, to compute upper limits to these quantities by assuming that the observed linewidth is dominated by one or the other of Eqns. 2 and 3.

To compute limiting values, we use linewidths of protonated carbons from the ${ }^{13} \mathrm{C}$ spectrum. For these carbons the dipolar coupling is overwhelmingly dominated by the directly bonded ${ }^{13} \mathrm{C}-{ }^{1} \mathrm{H}$ interaction due to the $r^{-6}$ term. The root mean square value of $\left(1-3 \cos ^{2} \theta^{\prime}\right)=0.91$ is used in Eqn. 3 . Limiting values for $\tau_{R, \ell}$ have been computed, based on a $\mathrm{C}-\mathrm{H}$ bond distance of $1.09 \AA$, and are given, along with measured linewidths, in Table II.

$\tau_{R, \ell}$ values for epinephrine range from $0.67\left(10^{-9}\right)$ to $2.0\left(10^{-9}\right) \mathrm{s}$ at $25^{\circ} \mathrm{C}$. These values may be compared to directly measured values of $\tau_{\mathrm{R}}$, based on NMR $T_{1}$ values [31], in a reference solution containing $0.5 \mathrm{M}$ epinephrine at 


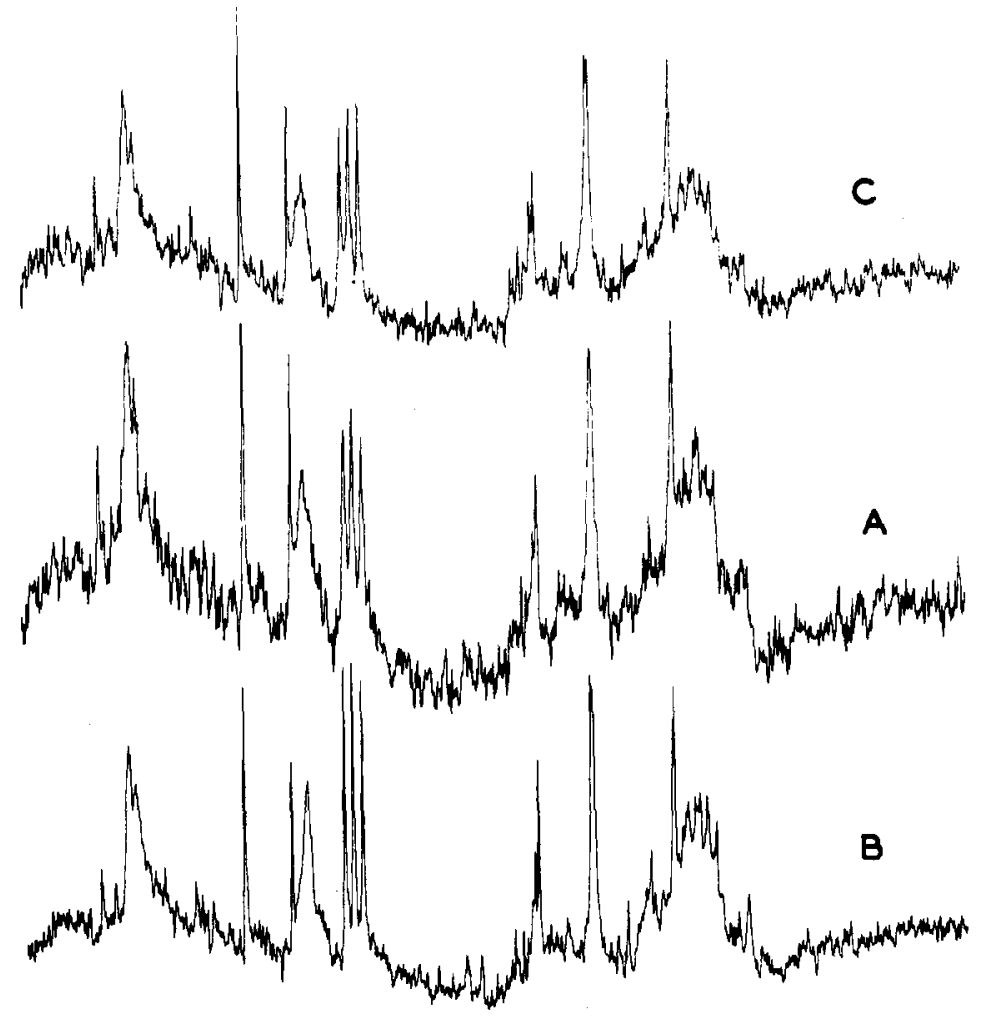

Fig. 4. Carbon-13 spectrum of chromaffin granules (a) on a sample prepared and maintained at $3^{\circ} \mathrm{C}$; (b) the same sample 30 min after the temperature has been raised to $25^{\circ} \mathrm{C}$; (c) the same sample after returning the temperature to $3^{\circ} \mathrm{C}$.

$\mathrm{p}^{2} \mathrm{H}$ 7. $\tau_{\mathrm{R}}$ for a given carbon in the reference solution is approx. 30 times shorter than computed values of $\tau_{R, \ell}$ in the granules. Since $\tau_{R}$ varies, in the Stokes-Einstein approximation [15], as the viscosity of the medium, the computed magnitude of $\tau_{\mathrm{R}, \ell}$ demonstrates that the intravesicular environment is fluid, although viscosities more than an order of magnitude greater than that

TABLE II

CARBON-13 LINEWIDTHS IN CHROMAFFIN GRANULES, AND LIMITING CORRELATIONS TIMES CONSISTENT WITH MEASURED LINEWIDTHS

\begin{tabular}{|c|c|c|c|c|}
\hline & \multicolumn{2}{|c|}{$T=3^{\circ} \mathrm{C}$} & \multicolumn{2}{|c|}{$T=25^{\circ} \mathrm{C}$} \\
\hline & $W(\mathrm{~Hz})$ & $\tau_{\mathbf{R}, \ell}(\mathbf{p s})$ & $W(\mathrm{~Hz})$ & $\tau_{\mathbf{R}, \ell}(\mathbf{p s})$ \\
\hline \multicolumn{5}{|l|}{ Epinephrine } \\
\hline $\mathrm{C}_{2}$ & 31 & 4.7 & 10 & 1.5 \\
\hline$c_{5}$ & 26 & 3.8 & 13 & 1.9 \\
\hline $\mathrm{C}_{6}$ & 33 & 4.9 & 10 & 1.5 \\
\hline $\mathrm{HCOH}$ & 24 & 3.4 & 10 & 1.5 \\
\hline $\mathrm{N}-\mathrm{CH}_{3}$ & $<21$ & $<1.0$ & $<14$ & $<0.67$ \\
\hline \multicolumn{5}{|c|}{ Chromogranin } \\
\hline Methylene & - & - & $\approx 25$ & $\approx 1.8$ \\
\hline$(\alpha-\mathrm{CH})$ & $<50$ & $<7.2$ & $<31$ & $<4.4$ \\
\hline
\end{tabular}


of water cannot be excluded by this calculation. On the other hand, the NMR linewidths show that the aqueous phase is essentially isotropic over regions quite large compared to molecular dimensions. Broadening in the granules is typically $20 \mathrm{~Hz}$, which implies limiting values of $\left\langle 3 \cos ^{2} \theta^{\prime \prime}-1\right\rangle \approx 0.001$. This small value could well be consistent with edge effects in a highly fluid aqueous pool several hundred ångstroms in diameter.

One of the most striking features of the NMR spectra is the approximate equivalence of the linewidths of the low molecular weight species and those of chromogranin, which is a fairly high molecular weight protein. (Smith and Winkler [12] report a molecular weight of 77000 for chromogranin A, the main soluble peptide.) In fact the carbon-13 spectrum of chromogranin in the granules contains lines of similar width in the methylene, methine region to those of quite small soluble proteins, such as native lysozyme $\left(M_{a}=14000\right)$ $[32,33]$, and it contains much more highly resolved structure than does the methylene region of native bovine serum albumin $\left(M_{A} 65000\right)$ [33]. This is true despite viscosity and/or anisotropy broadening that undoubtedly influences the granule spectra. The narrow linewidths of the protonated carbon peaks of chromogranin clearly point to a random coil protein that contains little or no secondary structure. An absence of secondary structure is especially evident in the narrow $\alpha-\left({ }^{13} \mathrm{CH}\right)$ peak, which reflects motion of the peptide backbone. It is also consistent with physical properties of isolated chromogranin reported by Smith and Winkler [12].

The insoluble lipid and protein fraction of the vesicles gives rise to a larger total proton NMR intensity than does the aqueous phase, although the lines are so broad that little of this intensity is apparent in high resolution spectra. The intensity of this ordered fraction is readily observed as the initial height of a free induction decay following a $90^{\circ}$ pulse [31], using a pulsed NMR spectrometer with short "dead time". Fig. 5 shows the rapid portion of the decay from a granule preparation that had been exhaustively exchanged with ${ }^{2} \mathrm{H}_{2} \mathrm{O}$ (99.6\%). Diode detection, which produces a voltage proportional to the absolute magnitude of the signal intensity, is used. A baseline trace is superimposed on the same photograph. The signal is composed of two components. The rapid component decays with a half-life of about $15 \mu \mathrm{s}$ and corresponds to an extremely broad resonance $\left(\Delta v_{1 / 2}=20 \mathrm{kHz}\right)$ in the frequency spectrum. This component evidently arises primarily from the ordered lipid phase. The decay is an order of magnitude more rapid than in simple lecithin bilayers [34] and is essentially at the "rigid lattice" limit, indicating that the chromaffin granule lipid phase is a relatively highly structured matrix.

\section{Models of chromaffin granule structure}

A number of structural models of chromaffin granules have been proposed since the early biochemical characterization by Hillarp, Blaschko and their coworkers. We consider here the most prominent of these models in the light of our NMR data.

(a) Protein gel hypothesis. Smith [9] has proposed that chromogranin forms a gel in the aqueous phase of the vesicle and that the gel holds epinephrine and ATP in an osmotically inactive form. In support of this hypothesis, Smith and Winkler [12] observed that isolated chromogranin gels spontaneously at $4^{\circ} \mathrm{C}$. 


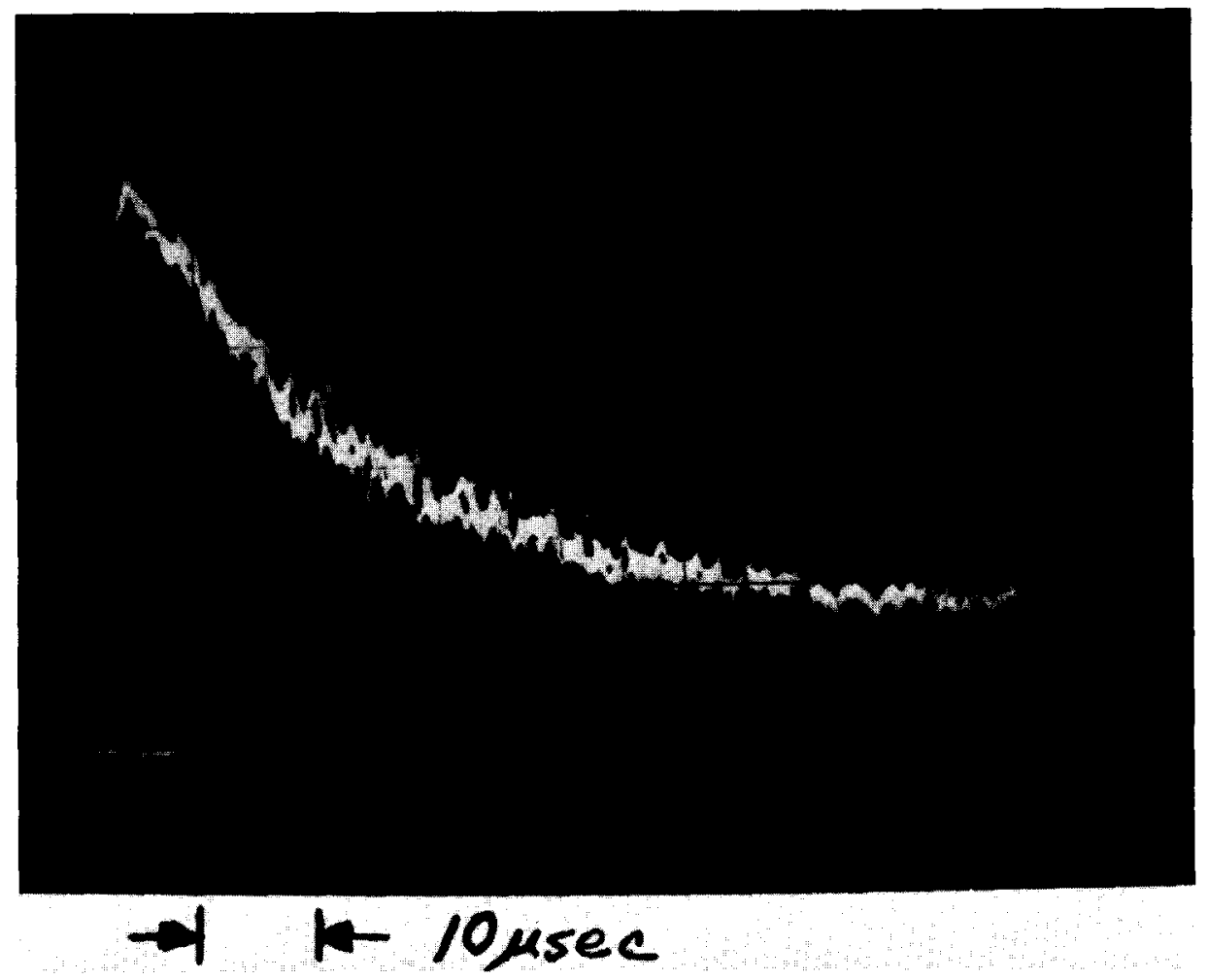

Fig. 5. Fast component of the free induction decay of protons $(54.1 \mathrm{MHz})$ in a sample of chromaffin granules at $3^{\circ} \mathrm{C}$. Diode detection with baseline shown on same exposure.

The presence of a gel in the aqueous phase of the granules is clearly ruled out by our NMR data however. A protein gel is generally defined as a phase that possesses rigidity due to a cross-linked three-dimensional protein network [35]. The local environment of a given peptide chain is necessarily anisotropic due to long-range order in the network. Consequently, very broad protein lines $\left(\Delta v_{1 / 2}\right.$ $>1 \mathrm{kHz}$ ) are expected in a gel, and NMR studies of ${ }^{1} \mathrm{H}_{2} \mathrm{O}$ relaxation in gelatin have shown that this is indeed true [25-27]. Chromogranin in the vesicles, on the other hand, is a random coil protein in a fluid, virtually isotropic environment. In his discussion of the gel hypothesis, Smith [9] has also suggested that a gel transition below about $10^{\circ} \mathrm{C}$ might account for the fact that the granules are stable indefinitely with respect to hormone efflux at low temperature $[4,6]$, while a portion of the endogenous epinephrine is labile at $20^{\circ} \mathrm{C}$ and above. No spectral changes indicative of gel transition are observed in the NMR spectra between -3 and $25^{\circ} \mathrm{C}$ however. A previous NMR study concluded that the vesicle interior is a "structured sol or gel" or "jelly" [1]. The term gel, which implies a protein network with long-range order, is in our view an unfortunate description since the NMR data are clearly inconsistent with such a phase.

(b) A lipoprotein matrix. Helle and Serck-Hanssen [36] have proposed that chromogranin is present as a lipoprotein matrix, which encloses tiny aqueous droplets, or channels, that are lined by hydrophilic sidechains of the protein. In 
this model, epinephrine and ATP are contained primarily in the aqueous phase, although epinephrine partitions also in the lipid phase. The NMR data, however, show that much, if not all of the chromogranin is dissolved in random coil form in the aqueous phase. Furthermore, the aqueous phase in sufficiently large to permit isotropic tumbling of the protein as well as of the smaller molecules. On the other hand, a large lipid fraction does exist and is present as a highly ordered phase, about which NMR gives little structural information,

(c) Binary and ternary complexes. Binary complexes involving ATP and epinephrine and ternary complexes involving ATP, epinephrine and chromogranin have been proposed to explain the anomalously low osmotic activity of the soluble components $[5,6,37-39]$. Binary epinephrine $\cdot$ ATP complexes have indeed been detected in aqueous solutions of these components [40-42]. Berneis et al. [43] found high molecular weight aggregates of ATP and epinephrine in the presence of divalent metal ions using ultracentrifugation techniques. Furthermore, ${ }^{31} \mathrm{P}$ spectra of the granules show that the ATP $\gamma$-phosphorus is shifted down field by $1.2 \mathrm{ppm}$ in intact vesicles. Daniels et al. [1] interpreted this shift as evidence for a binary epinephrine - ATP complex.

This kind of suggestive evidence may be misleading, however. We have noted above intensity differences in the ATP and epinephrine resonances which would not be expected if these components formed a simple binary complex. A comparison of NMR relaxation time (Sharp, R.R. and Richards, E.P., unpublished) more conclusively demonstrates the difference in correlation times for these molecules.

Although a binary complex is unlikely, epinephrine probably does participate in some form of complex that regulates its storage in the vesicles. Hillarp [6] has shown that while epinephrine diffuses freely through the chromaffin vesicle membrane at $4^{\circ} \mathrm{C}$, the endogenous pool of epinephrine is bound indefinitely in the presence of a concentration gradient. Even at $29^{\circ} \mathrm{C}$ about $80 \%$ of the endogenous epinephrine is maintained in the granules over a period of several hours [7]. Chromogranin, which is present as a random coil polyelectrolyte in the vesicles, may well provide a binding matrix for both ATP and epinephrine through polyelectrolyte-counterion interactions. Soluble polyelectrolytes exhibit very high binding affinity for their counterions through purely electrostatic attraction until the number of bound counterions provides approximate charge neutrality throughout the polyelectrolyte domain. Although Smith and Kirshner [44] found only weak complexes among the isolated soluble components, polyelectrolyte interactions may be quite subtle in intact chromaffin granules. The binding affinity between chromogranin and epinephrine depends strongly on the degree of neutralization of basic amino acid sidechains by ATP and on the presence of added metal ions. Interactions among the isolated soluble components appear to us to deserve further study.

\section{Conclusions}

The fact that the soluble components of chromaffin granules give rise to high resolution NMR spectra with reasonably narrow lines places important constraints on the phase structure of the vesicle interior. The narrow $\left(\Delta v_{1 / 2} \approx\right.$ $10-50 \mathrm{~Hz}$ ) lines observed show that ATP, epinephrine and chromogranin are 
dissolved in a fairly fluid aqueous phase that is essentially isotropic. Chromogranin appears to be a random coil protein with very little secondary structure. The NMR spectra conclusively rule out two previous hypotheses of chromaffin granule structure: (1) that the aqueous phase is a protein gel (Smith [9]) and (2) that the aqueous phase contains only epinephrine and ATP and is dispersed in narrow hydrophilic channels in a lipoprotein matrix (Helle and Serck-Hanssen [36]). The carbon-13 spectra also appear to be inconsistent with a simple binary epinephrine $\cdot$ ATP complex. The presence of chromogranin as a highly charged random-coil protein in the vesicles suggests that the storage complex may involve primarily electrostatic interactions between a polyelectrolyte and its counterions.

\section{Acknowledgement}

This work was supported in part by Institutional Research Grant No. IN-40P to University of Michigan from the American Cancer Society. Stimulating discussions with Dr. Edward Domino are gratefully acknowledged.

\section{References}

1 Daniels, A. (1974) Proc. R. Soc. Lond. Ser. B, 187, 353-361

2 Hillarp, N.-A. (1958) Acta Physiol. Scand. 43, 82-96

3 Smith, A.D. and Winkler, H. (1967) Biochem. J. 103, 483-492

4 Carlsson, A. and Hillarp, N.-Â. (1958) Acta Physiol. Scand. 44, 163-169

5 Hillarp, N.-A. (1958) Acta Physiol. Scand. 42, 321--332

6 Hillarp, N.-A, (1959) Acta Physiol. Scand. 47, 271-279

7 Hillarp, N.-A. (1958) Acta Physiol. Scand. 43, 292-302

8 Johnson, L.F. and Jankowsky, W.C. (1972) Carbon-13 N.M.R. Spectra, Wiley, New York, No. 356 (epinephrine); No. 384 (ATP)

9 Smith, A.D. (1968) in A symposium on the Interactions of Drugs and Subcellular Components in Animal Cells (P.N. Campbell, ed.) pp. 239-292, J. and A. Churchill, Ltd.

10 Stjärne, L. (1972) In Catecholamines (H. Blaschko, and E., Muscholl,) pp. 231-269, ed. SpringerVerlag, New York

11 Terland, O. and Flatmark, T. (1975) FEBS lett. 59, 52-56

12 Smith, A.D. and Winkler, H. (1967) Biochem. J. 103, 483-492

13 Roberts, G.C.K. and Jardetzky, O. (1970) Adv. Protein Chem. 24, 447-545

14 Carrington, A. and McLachlan, A.D. (1967) Introduction to Magnetic Resonance, Harper and Row, New York

15 Abragam, A. (1961) The principles of Nuclear Magnetism, Oxford University Press, Oxford

16 Slichter, C.P. (1963) Principles of Magnetic Resonance, Harper and Row, New York

17 Woessner, D.E. and Snowden, Jr., B.S. (1969) J. Chem. Phys. 50, 1516-1523

18 Woessner, D.E., Snowden, Jr., B.S. and Meyer, G.H. (1969) J. Chem. Phys. 51, 2968-2976

19 Woessner, D.E. and Snowden, Jr., B.S. (1969) J. Colloid Interface Sci. 30, 54-68

20 Woessner, D.E., Snowden, Jr., B.S. and Meyer, G.H. (1970) J. Colloid Interface Sci. 34, 43-52

21 Woessner, D.E. and Snowden, Jr., B.S. (1973) Ann. N. Y. Acad. Sci. 204, 113-123

22 Sterling, C. and Masuzawa, M. (1968) Makromol. Chem. 116, 140-147

23 Woessner, D.E. and Snowden, Jr., B.S. (1970) J. Colloid Interface Sci. 34, 290-299

24 Woessner, D.E. Snowden, Jr., B.S. and Chiu, Y.-C. (1970) J. Colloid Interface Sci. 34, 283-289

25 Kimmich, R. and Noack, F. (1970) Z. Naturforsch, 25A, 299-301

26 Kimmich, R. and Noack, F. (1970) Z. Naturforsch. 25A, 1680-1684

27 Outhred, R.K. and George, E.P. (1973) Biophys. J. 13, 83-103

28 Seiter, C.H.A. and Chan, S.I. (1973) J. Am. Chem. Soc. 95, 7541-7553

29 Horwitz, A.F. (1972) Membrane Molecular Biology (Fox, C.F., ed.), pp. 164-191, Sinauer Assoc., Stamford

30 Chapman, D. and Penkett, S.A. (1966) Nature 211, 1304-1305

31 Farrar, T.C. and Becker, E.B. (1971) Pulse and Fourier Transform N.M.R., Academic press, New York

32 Allerhand, A., Childers, R.F. and Oldfield, E. (1972) Biochemistry 12, 1335-1341 
33 Bradburg, J.H. and Norton, R.S. (1973) Biochim. Biophys. Acta 328, 10-19

34 Chan, S.I., Feigenson, G.W. and Seiter, C.H.A. (1971) Nature 231, 110-112

35 Ferry, J.D. (1948) Adv. Protein Chem. 4, 2-79

36 Helle, K.B. and Serck-Hanssen, G. (1975) Mol. Cell. Biochem. 6, 127-146

37 Blaschko, H., Born, G.V.R., D'lorio, A. and Eade, N.R. (1956) J. Physiol., Lond. 133, 548-557

38 Folck, B., Hillarp, N.-A. and Högberg, B. (1956) Acta Physiol. Scand. 36, 361-376

39 Helle, K.B. (1966) Mol. Pharmacol. 2, 298-310

40 Weiner, N. and Jardetzky, O. (1964) Arch. Exp. Pathol. Pharmakol. 248, 308-318

41 Tuck, L.D. and Baker, J.K. (1973) Chem. -Biol. Interactions 7, 355-366

42 Maynert, E.W., Moon, B.H. and Pai, V.S. (1970) Mol. Pharmacol. 8, 88-94

43 Berneis, K.H., Pletcher, A. and DaPrada, M. (1969) Nature 224, 281-282

44 Smith, W.J. and Kirshner, N. (1967) Mol. Pharmacol, 3, 52-62

45 Keim, P., (1973) J. Biol. Chem. 248, 6104-6113

46 Keim, P., (1973) J. Biol. Chem. 248, 7811-7818

47 Keim, P., (1974) J. Biol Chem. 249, 4149-4156

48 McDonald, C.C. and Phillips, W.D. (1969) J. Am. Chem. Soc. 91, 1513-1525

49 Christl, M, and Roberts, J.D. (1972) J. Am. Chem. Soc. 94, 4565--5473

50 Stothers, J.B. (1972) Carbon-13 NMR Spectroscopy, Academic Press, New York 